\title{
Line focusing for soft x-ray laser-plasma lasing
}

\author{
Davide Bleiner, ${ }^{*}$ Jürg E. Balmer, and Felix Staub \\ Institute for Applied Physics, University of Bern, Sidlerstrasse 5, CH-3012 Berne, Switzerland \\ *Corresponding author: bleiner@iap.unibe.ch
}

Received 17 June 2011; revised 23 September 2011; accepted 28 September 2011; posted 29 September 2011 (Doc. ID 149400); published 20 December 2011

\begin{abstract}
A computational study of line-focus generation was done using a self-written ray-tracing code and compared to experimental data. Two line-focusing geometries were compared, i.e., either exploiting the sagittal astigmatism of a tilted spherical mirror or using the spherical aberration of an off-axisilluminated spherical mirror. Line focusing by means of astigmatism or spherical aberration showed identical results as expected for the equivalence of the two frames of reference. The variation of the incidence angle on the target affects the line-focus length, which affects the amplification length such that as long as the irradiance is above the amplification threshold, it is advantageous to have a longer line focus. The amplification threshold is physically dependent on operating parameters and plasma-column conditions and in the present study addresses four possible cases. (c) 2011 Optical Society of America OCIS codes: $140.0140,140.7240,350.5400$.
\end{abstract}

\section{Introduction}

A high irradiance laser's focal spot, shaped as a narrow line of $10-20 \mathrm{~mm}$ in length by $10-50 \mu \mathrm{m}$ in width, induces the formation of a plasma column above the irradiated target. The plasma column is functional to the generation of an extreme ultraviolet (EUV; i.e., $\lambda \sim 10-50 \mathrm{~nm})$ or soft $\mathrm{x}$-ray $(1-10 \mathrm{~nm})$ coherent radiation (laser) by means of amplified spontaneous emission (ASE) in single-pass amplification along the column's length. Besides cylindrical lenses, parabolic and spherical reflectors have proved efficient in generating a line focus, i.e., utilizing either (i) astigmatism or (ii) spherical aberration. Astigmatism is observed when the tangential and sagittal foci do not coincide, which induces two line foci on the corresponding planes. A tilt of the focusing mirror, centrally illuminated, leads to a change of the tangential focus as given by

$$
f_{T}=(R / 2) \cos \theta
$$

and a change of the sagittal focus as given by

0003-6935/11/366689-08\$15.00/0

(C) 2011 Optical Society of America

$$
f_{S}=(R / 2) / \cos \theta,
$$

where $R$ is the radius of curvature of the mirror and $\theta$ is the mirror tilt angle. The focal spots thus appear stretched over a certain length as a thin stripe of light. On the other hand, spherical aberration is the variation of focus location with illumination aperture on the sphere surface. A spherical mirror can thus induce a line focus stretched along the focal plane when illuminated nonradially with a pencil of light impinging on an off-axis region. In the particular case of an illumination beam parallel to the mirrorsymmetry axis, the line focus is produced along such an optical axis itself. The general case of nonparallel orientation has been discussed elsewhere [1,2].

The two frames of reference while generating a line focus, i.e., that of exploiting astigmatism (on-axis illumination of a tilted reflector) and that of using spherical aberration (off-axis illumination of a reflector), are physically equivalent and produce the same irradiance distribution on the target. This is mathematically proved if a reference system transformation is deployed. Figure 1 shows the equivalence between astigmatic focusing and spherical-aberration focusing. The former geometry implies tilting a reflector (thick traces in Fig. 1) that can be interpreted as a segment of a large-aperture reflector (thin traces in Fig. 1) of 


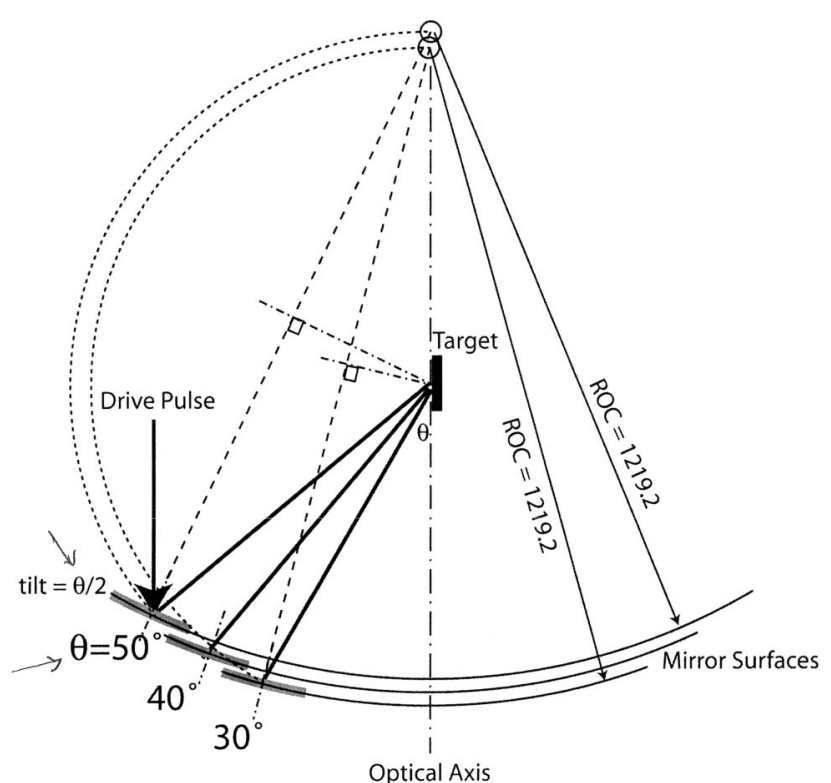

Fig. 1. Geometry of the line-focus generation with a spherical reflector oriented for three grazing incidence angles on the target. In the sketch the target position is kept fixed, and the reflector is moved correspondingly. The radius of curvature (ROC) is taken as in the Bern x-ray laser facility ("BeAGLE" system). The sketch visualizes the equivalence between the two discussed line-focusing geometries; i.e., thick traces indicate tilted reflectors and thin traces indicate an off-axis large-aperture illuminated reflector.

identical radius of curvature. In Fig. 1 , the line focus lies along the optical axis, and in order to keep the target fixed in space, the spherical collectors are shifted accordingly. In fact, to keep the target fixed at the center of Fig. 1, the tilted mirror is shifted backward for decreasing grazing angle $(\theta)$ according to the distance of the sagittal focus.

In x-ray laser science there is an interest in generating line foci at a low grazing angle to maximize the output by means of so-called grazing incidence pumping (GRIP) [3]. On the other hand, a large grazing angle is beneficial for scaling down the output wavelength. In fact, due to the $\sin ^{2} \theta$ dependence of the optical penetration depth into a plasma [4], a large $\theta$ permits depositing of the energy deeper into the plasma, at higher electron densities, which is required for short-wavelength lasing. Hence a first issue to address while line focusing is the trade-off between efficiency and wavelength scaling.

Furthermore, the use of focusing reflectors has shown advantages in terms of intensity throughput, lower wavefront distortion, and damage threshold with respect to cylindrical lenses. A parabolic mirror is beyond the scope of the present communication. Our investigations indicated that a parabolic mirror produces broader line foci than an equivalent spherical mirror. A broad line focus can make the overlapping of prepulses to the main pulse easier. On the other hand, spherical mirrors may produce significantly hotter and denser plasma conditions at comparable drive beam energy by means of tighter focusing.
The aim of this work was to investigate the parameter-dependent characteristics of the line focus obtained with a spherical reflector and relate them to the laser plasma column for ASE. More specifically, the study considered the angle of incidence, the profile of the driver beam, and the beam diameter. We will show results obtained with an inhouse code and validate the computational results with previous experimental data [5].

\section{Methods}

Simulations were done using self-written threedimensional (3D) ray-tracing code (in the following just KuBERT from "Kubisch BErn Ray Tracing"). KuBERT has two major advantages: 1) it is rapid, both in the geometry construction and in the problem solving, since it focuses on computation of the very essential information we are interested in; 2) it is self-written, and hence open-source and more manageable and controllable in its functionality. Its capability for vectorial propagation of arbitrary beam profiles in optical media makes it an effective tool for the study of line-focus irradiance distributions as a function of beam profile and focusing geometry. The Visual Basic code (based on the .NET Framework library) takes a number of inputs for the illumination (e.g., ray bundle discretization and width, divergence, energy, wavelength) and for the optics, such as element type (e.g., lens, mirror, grating,), vertex coordinates, shape, rotation, and offset. Special illumination profiles (e.g., "super-Gaussian") can be realized using masks in front of the homogeneous source. Given a certain optics type, additional inputs may be requested, such as refractive index for lenses. The optics are thus modeled as sequential objects, rather than as juxtaposed surfaces as in much commercial software. This facilitates the construction of the geometry. The input data are processed as a vector basis (multiplied by the given energy distribution function) using free beam propagation, Snell's law, and reflection law, finally counting the ray-trace intercepts on an autofocus plane (image). This permits us to generate a number of plots, such as ray path, intensity profiles (one-dimensional) or mappings (two-dimensional) at given surfaces, spot diagrams (vector propagation), optical path length, and traveling-wave speed (useful for x-ray laser studies). No physical effects are accounted for (only geometry), such as polarization, interference, etc. The geometric propagation is in $3 \mathrm{D}$.

The driver laser was simulated as a superGaussian beam, i.e., characterized by a radial intensity profile given by the following relation:

$$
I(r)=I_{o} \operatorname{Exp}\left[-2\left(r / r_{o}\right)^{2 N}\right],
$$

where $I_{o}$ is the peak intensity, $r_{o}$ is the beam radius, and $N$ is the super-Gaussian exponent that for $N=1$ provides a TEM $\mathrm{T}_{00}$ mode profile. As given in Table $\underline{1}$, the default value was $N=6$. 
Table 1. Parameter Settings

\begin{tabular}{lcc}
\hline Parameter & Default Value & Range of Study \\
\hline Angle of incidence & $45^{\circ}$ & $25^{\circ}-55^{\circ}$ \\
Mirror shape & Spherical & Fixed \\
Beam diameter & 80 & $20-100$ \\
Mirror radius & 1219.2 & Fixed \\
Beam profile & Super-Gaussian, & $N=1,2,10$ \\
[Eq. ( [ $)$ [ & $N=6$ & \\
\hline
\end{tabular}

The driver beam was delivered with a $45^{\circ}$ turning mirror toward a focusing spherical reflector whose radius of curvature was $1219.2 \mathrm{~mm}$, i.e., a focal length of $f=609.6 \mathrm{~mm}$ ( 24 inches). The different focusing geometries were simulated as shown in Fig. $\underline{2}$. In the astigmatic line focus ("A"), the reflector was a spherical mirror that was tilted to the incoming laser beam [Fig. 2(a)]. The tilt angle was half the incidence angle on the target. The position of the target was chosen in order to minimize the width of the sagittal focus in agreement with Eq. (2). The orientation of the sagittal focus lies perpendicular to the plane of the page. The line focusing using spherical aberration ("B") was simulated with the same spherical reflector radius of curvature (ROC) as in "A", i.e., $\mathrm{ROC}=1219.2 \mathrm{~mm}$, but nontilted, and whose optical axis was coincident with the target orientation [Fig. 2(b)]. The reflector was illuminated parallel to the optical axis at an off-axis distance corresponding to specific incidence angles on the target.

Benchmarking data were available from a previous study. As explained in Ref. [5], these were acquired using a modified target mount to allocate a CCD detector. The laser was delivered at $45^{\circ}$ and attenuated using the transmission of a highly reflecting plane mirror followed by neutral density filters. The measurements are combined from several images taken at different camera positions along the line focus, as the detector width was smaller than the length of the focal line.

\section{Results \& Discussion}

\section{A. Parametric Optimization}

Figure 3 summarizes the parametric study for a line focus produced exploiting astigmatism ("A") and spherical aberration ("B"). The plots consistently show the physical equivalence of the line-focus irradiance distributions under the two frames of reference. These irradiance distributions were generated under different parameter configurations, as explained below, where the area was kept constant at $1 \mathrm{~J}$ pulse energy. In Fig. 3 (top) one can observe the effect of the incidence angle on the irradiance distribution and line-focus length. The profiles become shallower with increasing incidence angle. The fact that the energy is stretched over a longer line length at increasing incidence angle explains the reduction of peak irradiance (signal integrals are constant). Figure 3 (middle) shows the influence of the driver beam profile indicated as an exponent of the superGaussian profile. One notices that the higher the super-Gaussian order, the steeper the profile is. Finally, Fig. $\underline{3}$ (bottom) shows the effect of the beam width. It is evident that the line-focus length scales up with the beam diameter. Narrow beams lead to higher irradiation peaks as a consequence of the concentration of the delivered energy over shorter linefocus lengths. This effect is indeed complementary to that of the incidence angle.

It is noteworthy to compare the obtained line-focus lengths, computed under these configurations, with existing analytical models. Ref. [6] has derived a trigonometric expression for the line-focus length $(L)$ as a function of grazing angle $(\theta)$ and beam width $(d)$, with the reflector focal length $(f)$ as a control parameter, which is as follows:

$$
L=\frac{f}{\sqrt{1-\left(\sin \vartheta+\frac{d}{4 f}\right)^{2}}}-\frac{f}{\sqrt{1-\left(\sin \vartheta-\frac{d}{4 f}\right)^{2}}} .
$$

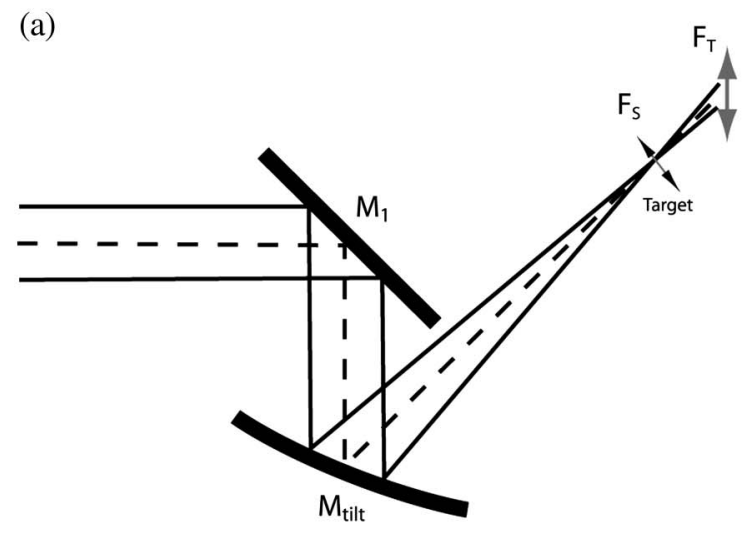

(b)

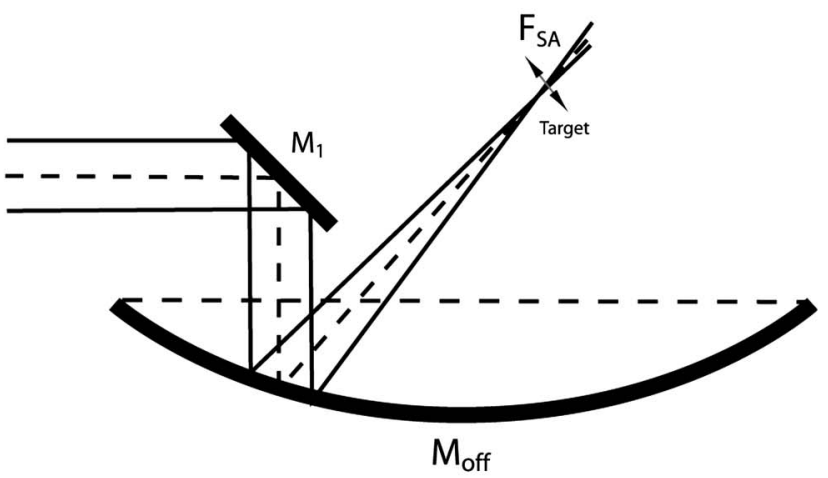

Fig. 2. Schematic of the focusing reflectors, here for the case of a $45^{\circ}$ grazing angle. (a) Astigmatic line focus by tilted mirror ( $\mathrm{M}_{\text {tilt }}$ ) with tangential focus $\left(\mathrm{F}_{\mathrm{T}}\right.$, length parallel to the page) and sagittal focus ( $\mathrm{F}_{\mathrm{S}}$, length normal to the page) at the target position; (b) off-axis illumination of the nontilted mirror $\left(\mathrm{M}_{\text {off }}\right)$ to exploit its spherical aberration for a line focus $\left(\mathrm{F}_{\mathrm{SA}}\right)$ at the target position. 

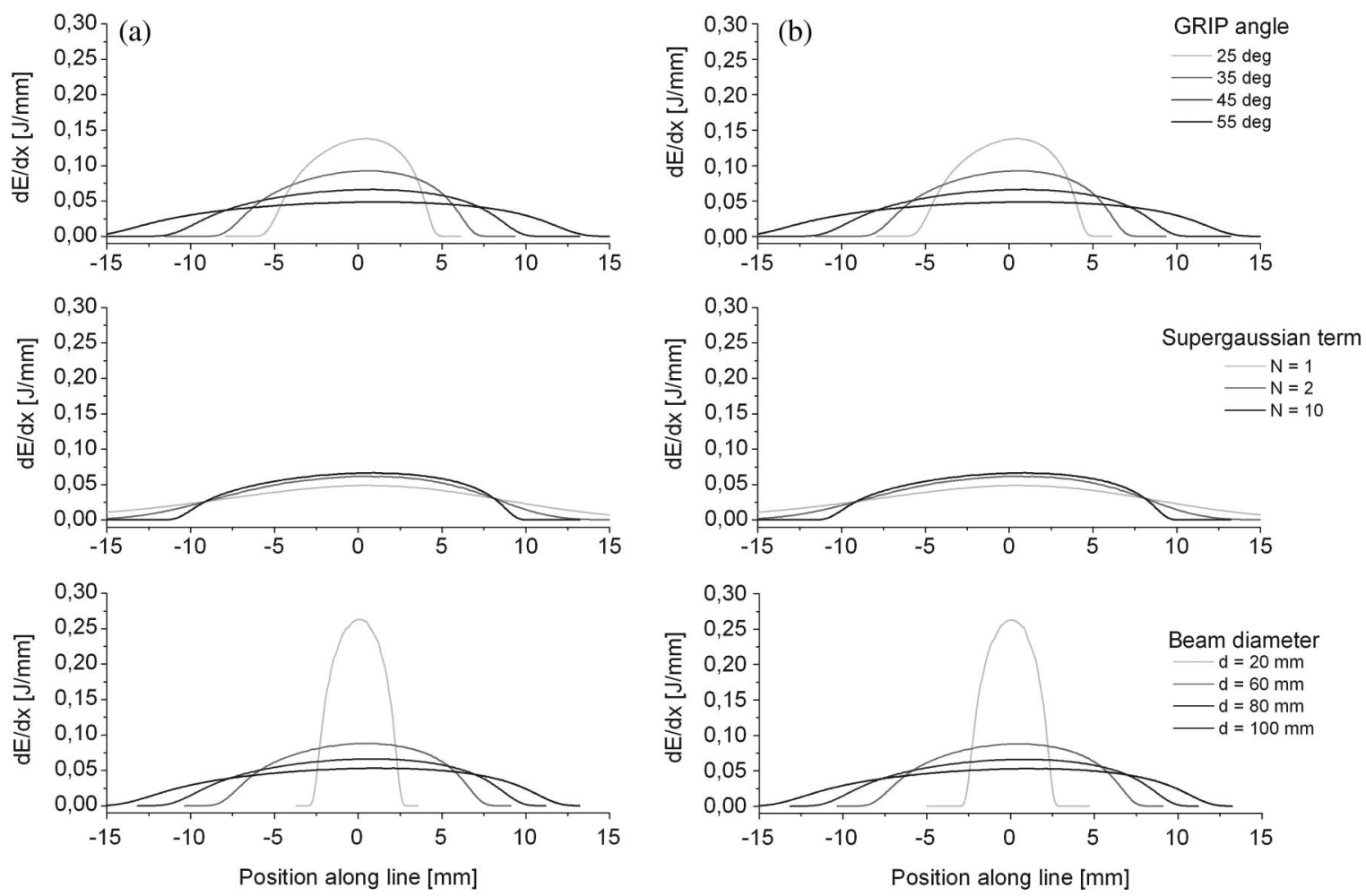

Fig. 3. Calculated line-focus profiles produced by means of (a) sagittal focus with tilt-induced astigmatism and (b) off-axis spherical aberration, as a function of incidence angle (top), driver beam spatial profile (middle), and driver beam diameter (bottom).

The predicted line-focus length is consistent with what is obtained with our ray-tracing computations. Figure 4 shows such a comparison between the ray tracing and the analytical model. Figure $4(\mathrm{a})$ shows the dependence on the incidence angle. The ray-tracing code provides consistent results among the two focusing geometries. Figure 4(b) shows the dependence on the beam width. Analytical results from Ref. [6] and our ray tracing are slightly mismatched because the analytical expression refers
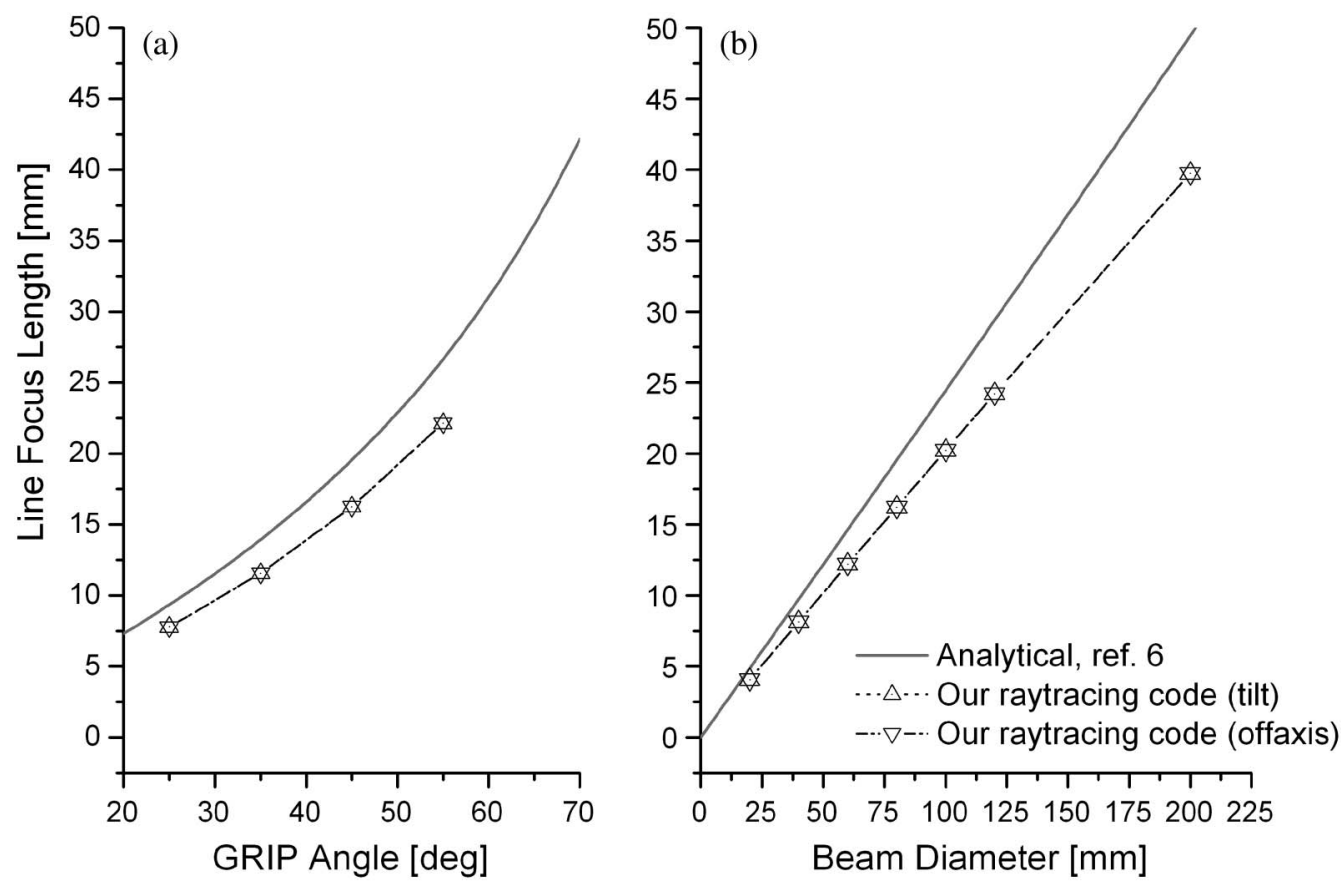

Fig. 4. Line-focus length (a) as a function of incidence angle at $80 \mathrm{~mm}$ diameter and (b) as a function of beam diameter for $45^{\circ}$ incidence, determined with various computational methods discussed in the text. 
to the full line length on the target surface and also assumes a fully flat-top profile. In our ray-tracing computation we have more fully accounted for physical factors, such as the illumination profile (superGaussian), the intensity distribution on the target, and the geometry of focusing.

The irradiance distributions in Fig. 3 show that by changing one parameter, multiple profile characteristics are modified. For instance, an increase in incidence angle alters the leading and the trailing edges, the overall length, etc. For small beam diameters the profile structure is not changed but only scaled, except when changing the beam profile. With increasing beam diameter the line-focus profile becomes increasingly asymmetric. To highlight such interparametric dependence, normalization of both FWHM and peak were applied to the distributions, from the data shown in Fig. $\underline{3}$ and visualized in Fig. $\underline{5}$. In these plots the FWHM is centered on 0 (i.e., from -0.5 to 0.5 ), and the irradiance values of each curve are taken from the background-corrected value. In physical terms, this corresponds to changing pairs of parameters consistently to maintain the linefocusing extension. For instance, one may cochange the incidence angle and beam width altogether, such that the line-focus length remains constant at various incidence angles. One notices that the distributions for the case of the variation of the incidence angle (Fig. $\underline{5}$, top) and the variation of beam diameter
(Fig. 5, bottom) show insignificant modification. This indicates that the irradiance structure is not modified by operating on such parameters; only the distributions are rescaled to different extents. The change of the beam Gaussian profiles is, on the other hand, causing a significant remodulation in distribution bandwidth, especially noticeable at the edges (Fig. 5, middle). This suggests that one can consistently optimize the line-focus irradiance profiles by operating on angle and beam width and preferably homogenizing the driver beam profile (e.g., superGaussian $N>6$ ).

\section{B. Amplification Length}

The line-focus optimization affects the driver irradiance as well as the effective amplification length. The driver irradiance must be higher than a given amplification threshold, but indeed not much higher to prevent loss of inversion due to collisional deexicitation in an overly hot/dense plasma [7]. Any excess beam energy, with respect to the threshold requirements, may be instead used for extending the line-focus length. Increasing the line-focus length as much as possible is advantageous for increasing the amplification length, as long as the pump irradiance is above the threshold value. The threshold value can be inferred from the optimum plasma temperature/density for EUV amplification [8].
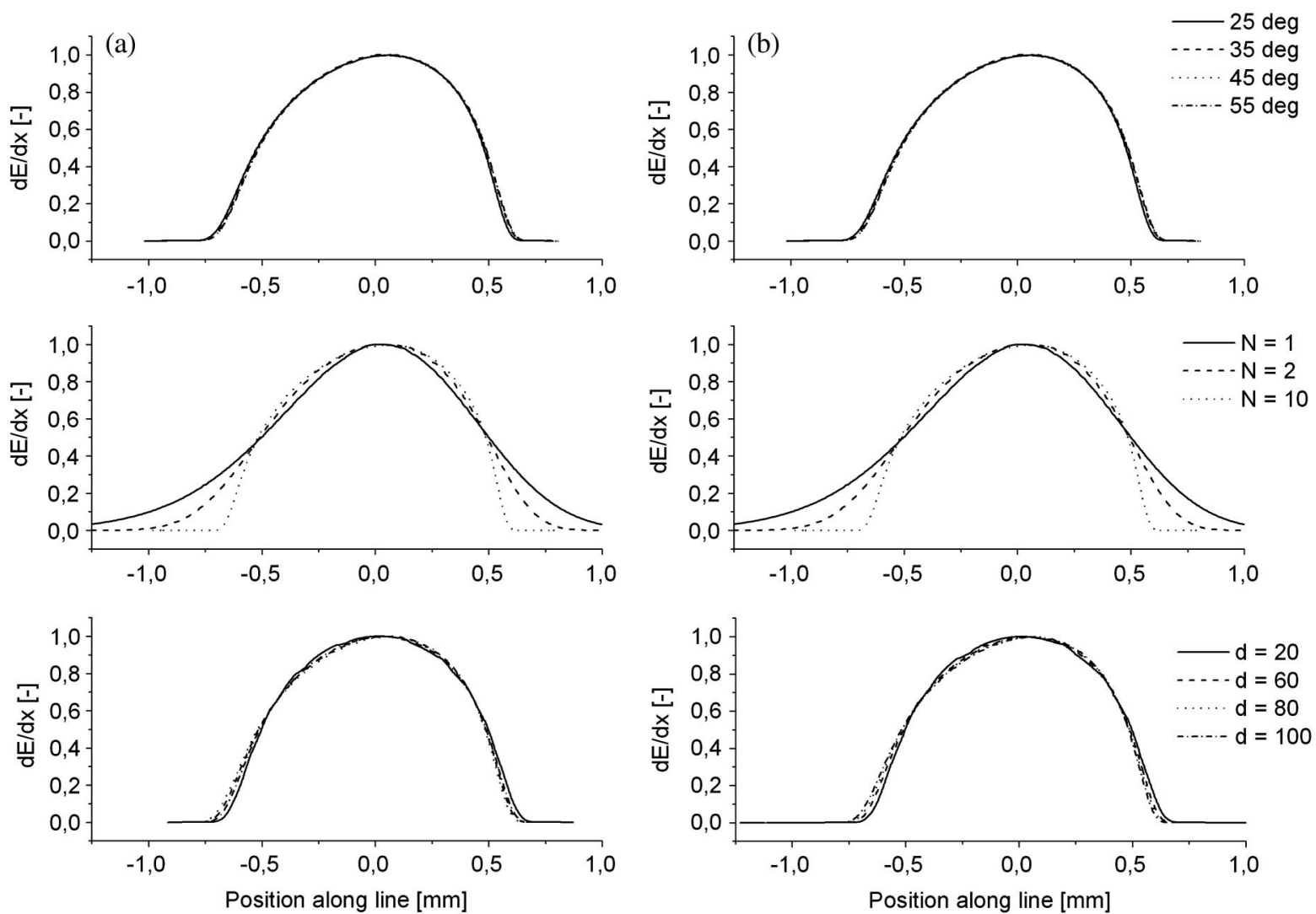

Fig. 5. Width- and peak-normalized line-focus irradiance profiles produced by means of (a) sagittal focus with tilting-induced astigmatism and (b) off-axis spherical aberration as a function of incidence angle (top), beam profile (middle), and beam diameter (bottom). 
The line-focus length can be increased, as shown above, with a larger grazing angle, as long as the decrease in peak irradiance is still above the amplification threshold. The increase of the grazing angle should thus produce increasingly stronger $x$-ray laser amplification, because a noise signal above threshold is amplified over a longer column. For an excessive increase of the grazing angle, the peak irradiance along the line focus drops beneath the threshold value and thus impedes noise signal growth in an ASE regime.

Analyzing the structure of the line-focus irradiance distributions shown in Fig. 3(a), one notices that two incidence curves cross each other at specific points on their trailing and leading edges. The leadingedge cross point (let us call it "L") is higher than the trailing-edge one (let us call it "T"), as shown in Fig. 6(a). This is due to the skewness of the profiles. Considering that the skewness is gradually mitigated at increasing incidence angle, the height gap between the two cross points also is consistently reduced at a large grazing angle.

Let us consider, as visualized in Fig. 6(a), a horizontal line that indicates the amplification threshold. The amplification length is thus the segment joining the incidence curve trailing and leading edges above the threshold line. If such a threshold is very low, all irradiance curves shown are mainly in the amplification range. If the threshold is elevated, the shallowest curve will gradually fall below the amplification cutoff. More specifically, one can define
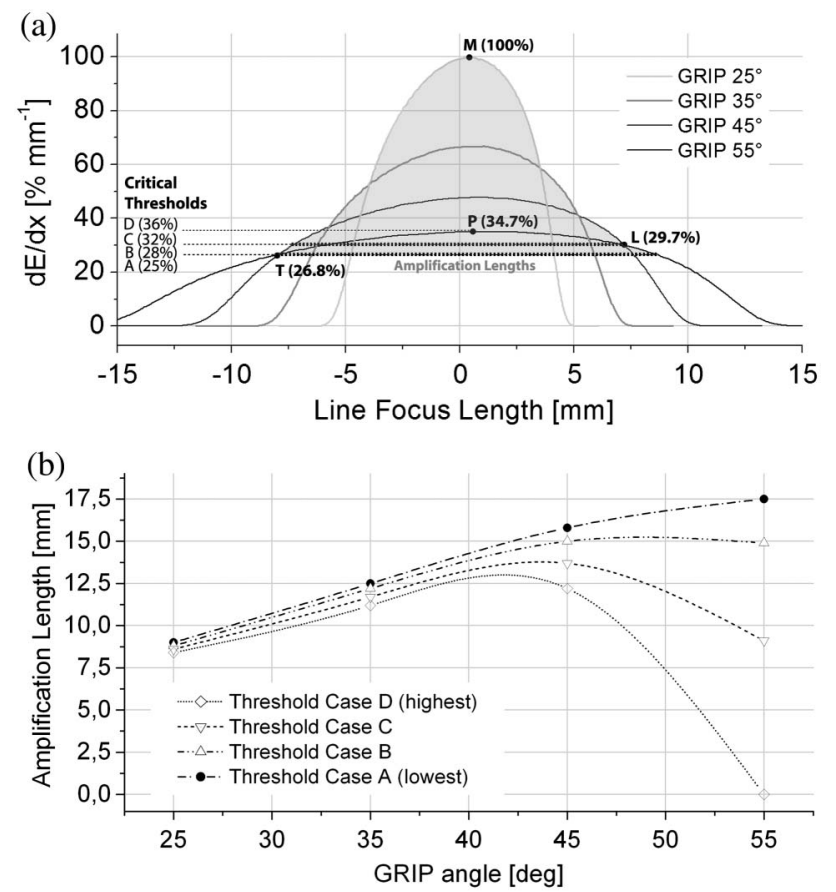

Fig. 6. (a) Normalized line-focus irradiance distributions as a function of incidence angle from Fig. 3(a). One can define four important cases for an amplification threshold, as explained in the text (cases are labeled as A, B, C, D). (b) Depending on the four cases, the amplification length will have an optimum (high threshold) or not (low threshold), as a function of incidence angle. four important cases depending on the height of the threshold, namely:

Case A: The threshold is at the point $\mathrm{T}$ (lower cross point).

Case B: The threshold is between point $\mathrm{T}$ and point $\mathrm{L}$ (higher cross point).

Case $C$ : The threshold is between point $\mathrm{L}$ and point $\mathrm{P}$ (curve peak).

Case D: The threshold is at or above P.

If the amplification threshold is consistent with case $\mathrm{A}$, then the irradiance is above the amplification threshold even at the largest possible incidence angle. Figure 6(b) shows the amplification length as a function of grazing angle for the four cases. The discussed case A has no definitive optimum (top value), since the threshold is the lowest and any irradiation induces amplification. If the amplification threshold fits case B, the amplification length tends to reach a plateau at a large grazing angle [Fig. 6(b)]. If the threshold is further raised to fit case $C$, the amplification length increases with increasing grazing angle up to an optimum value. Hence, the occurrence of an optimum value in the amplification length versus the grazing angle dependence is indicative of a threshold located within the range of the irradiances deposited on the target. Finally, if the threshold fits case $\mathrm{D}$, the amplification length will increase at a comparable rate, as in the cases above [Fig. 6(b)], but beyond a certain optimum value, the drop of amplification length is more pronounced. Hence, the higher the threshold, the faster the drop is at high grazing angles, as shown in Fig. 6(b).

The model presented above is in agreement with our own experimental observation published previously [5] concerning the occurrence or lack of an optimum, which was so far not fully understood. In that work we indeed reported experimentally the absence/occurrence of an optimum for the case of palladium $(\mathrm{Pd})$ target irradiated with $2 \mathrm{~J}$ and $5 \mathrm{~J}$. The irradiance distribution profiles obtained at $2 \mathrm{~J}$ and a large grazing angle were possibly closer to the amplification threshold, which is confirmed by an optimum value at $\theta=35^{\circ}$. In the case at $5 \mathrm{~J}$, all studied irradiance profiles were largely above the amplification threshold, which did not provide any specific optimum up to the boundary data.

For the cases analyzed here, i.e., with the set of curves at incidence angles of $25^{\circ}, 35^{\circ}, 45^{\circ}$, and $55^{\circ}$, we have used the $\theta=25^{\circ}$ peak height (point M) as the normalization value and computed the other curves accordingly in a percent scale [Fig. 6(a)]. In this respect, the $\mathrm{T}$ and the $\mathrm{L}$ points are identified as crossing points among the $\theta=45^{\circ}$ and the $\theta=$ $55^{\circ}$ curves at ordinate values as follows: $\mathrm{T}$ is at $26.8 \%, \mathrm{~L}$ is at $29.7 \%, \mathrm{P}$ is at $34.7 \%$. The discussion here is based on four arbitrarily chosen irradiance curves, but it can be generalized for any set of $N$ irradiance distributions and the crossing between any arbitrarily spaced $N$ th and $(N-1)$ th irradiance 
curves. The discussion here neglects refractive losses and assumes that a longer plasma column implies a longer gain-length product.

C. Incidence Angle Adjusted by Tilting the Target

Grazing angle variation has been proposed by tilting the target instead of moving the focusing optics [9]. The characteristics of the line focus under these focusing conditions are therefore investigated. Although such an inclination of the target correlates with the incidence angle, it should be noticed that the beam profile typically evolves along the direction of propagation. At the sagittal focus a line focus is "naturally" generated. The latter evolves in the sagittal and tangential planes, such that the aspect ratio is inverted and a $90^{\circ}$ rotated line spot is produced at the tangential focus. By tilting the sample, additional aberration and coma are induced. The spot diagrams obtained for the four incidence angles discussed above are shown in Fig. 7 , with a tilt angle of the target of $2^{\circ}$ with respect to the nominal value.

The spot diagrams of Fig. 7 are indeed scaleinvariant, and when plotted nondimensionally they look identical. The most distinguishing feature is the bilobed ("propellerlike") shape, which indicates a variation of focal irradiance across the spot. The trailing and leading edges are thus characterized by lower irradiance than the central part. This effect
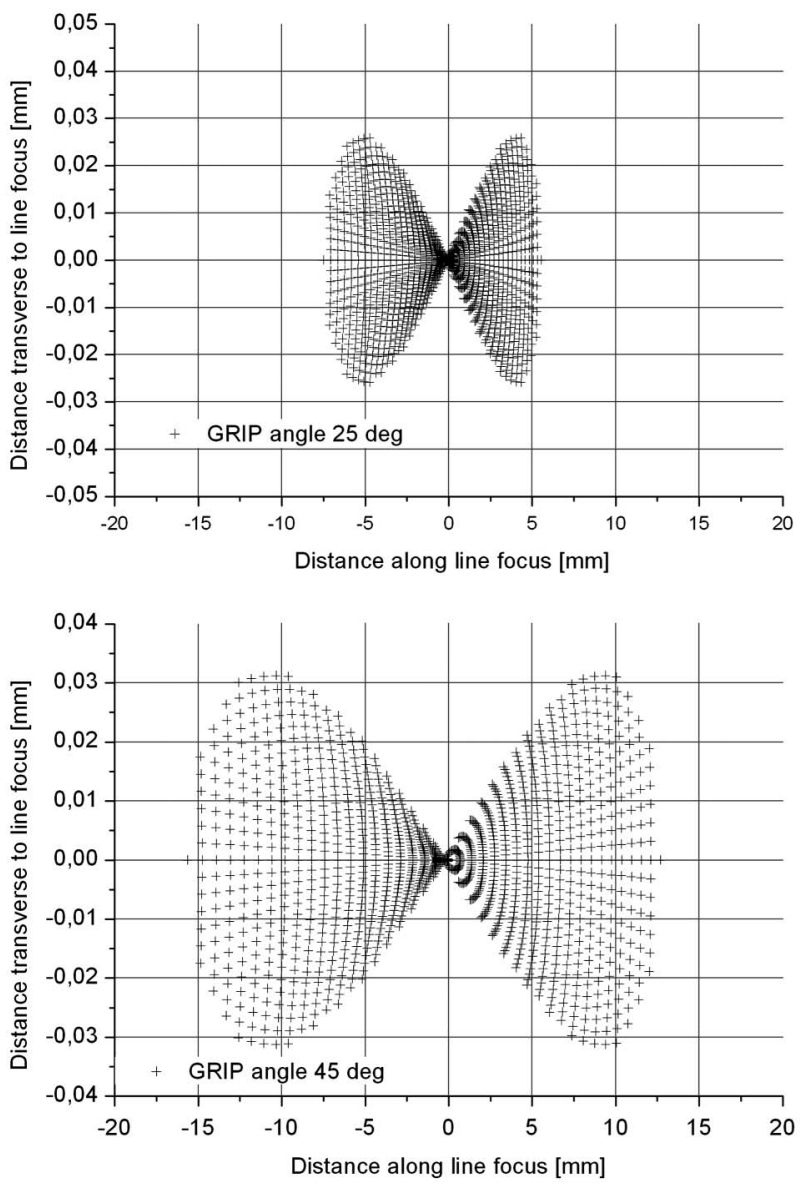

is exacerbated at large grazing angles, with the conclusion that at large target tilt the plasma column is characterized by increased longitudinal gradients in temperature and density.

In Ref. [9], these effects are not observed for the specific experimental setup chosen. In fact, the target was tilted over a relatively wide angular range of $12^{\circ}$, while the grazing angle on the focusing mirror was kept constant at $7^{\circ}$. An adjustable cylindrical lens before the compressor was used to keep the length of the line focus constant and to avoid the formation of a bilobed line focus.

\section{Ray-Tracing Validation}

Having so far compared computational results, e.g., of analytical versus ray-tracing simulations, we turn now to a comparison with the experimental results (Fig. 8). We show the specific case of a $45^{\circ}$ angle of incidence and a beam of $60 \mathrm{~mm}$ (FWHM) in diameter, which is what our experimental setup permitted us to acquire. One notes that our ray-tracing code captures the profile quite faithfully throughout. The measurement was unfortunately affected by high frequency noise and lower frequency fluctuations that are smoothed out computationally. The predictive value of our computation is, however, validated, and with it the extent of the implications discussed
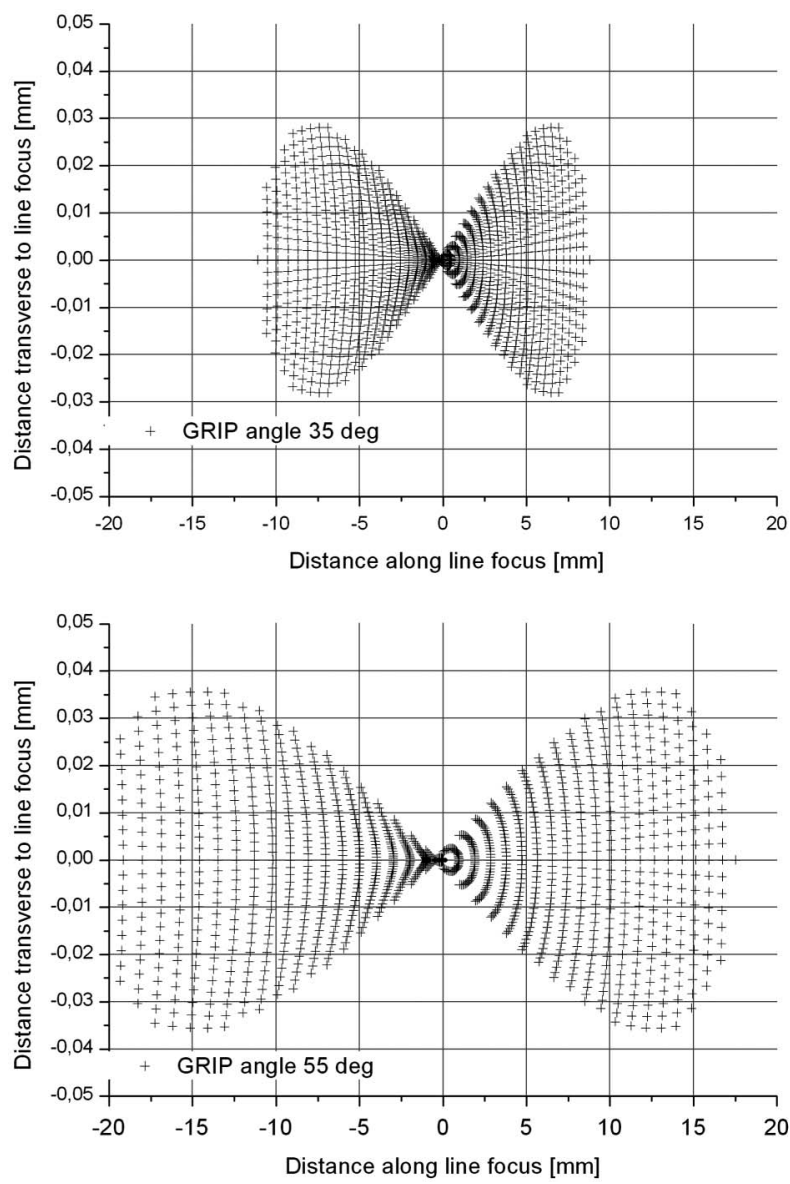

Fig. 7. Spot diagrams obtained by ray tracing with an additional target tilt of $2^{\circ}$ to obtain the indicated incidence angles. 


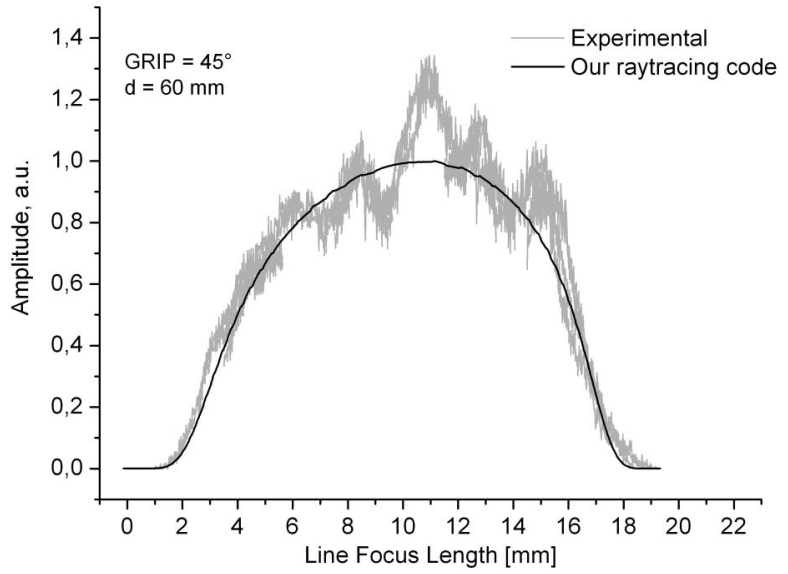

Fig. 8. Comparison of experimental and computational results. The experimental curve was obtained with an incidence angle of $45^{\circ}$, a beam diameter of $60 \mathrm{~mm}$ (FWHM), and a super-Gaussian profile of order $N=6$.

for optimized line focusing in soft x-ray amplified spontaneous emission lasing.

\section{Conclusions}

Line-focus irradiation is important for the generation of a plasma column to sustain ASE for laboratoryscale soft x-ray lasers. The adjustment of the drive beam parameters and irradiation scheme is important to obtain saturated output, especially at a short wavelength, which demands conflicting conditions in terms of GRIP angle. In this work we performed a computational study of line-focus generation using self-written ray-tracing software and comparing the obtained results to experimental measurement of the line focus. For the calculations, two beam focusing approaches were compared, i.e., (1) exploiting astigmatism of a tilted spherical reflector and (2) using spherical aberration from off-axis spherical mirror illumination. In both cases the line-focus length was shown to increase with a larger grazing incidence angle, in agreement with the literature data. Computed irradiance distributions were shown to have a close match with experimental data of line-irradiance profiles. We demonstrated that the amplification length is optimized at the largest grazing angle for which the irradiance is still above the amplification threshold. Therefore, a "sledge-hammer approach," i.e., high peak irradiation at short line foci, may lead to nonoptimum x-ray output. The delivering of the drive pulses at the incidence angle induced by tilting the target, instead of the focusing setup, was shown to deteriorate the line focus characteristics.

The authors are grateful to T. Feurer for invaluable support and to C. Imesch and M. Grünig for preliminary investigations. The project is supported by the Swiss National Science Foundation and partly by the Holcim Stiftung Wissen. Discussions with participants of the COST MP0601 actions have been also useful.

\section{References}

1. I. N. Ross and E. M. Hodgson, "Some optical designs for the generation of high quality line foci," J. Phys. E 18, 169-173 (1985).

2. I. N. Ross, J. Boon, R. Corbett, A. Damerell, P. Gottfeldt, C. Hooker, M. H. Key, G. Kiehn, C. Lewis, and O. Willi, "Design and performance of a new line focus geometry for x-ray laser experiments," Appl. Opt. 26, 1584-1588 (1987).

3. R. Keenan, J. Dunn, V. N. Shlyaptsev, R. F. Smith, P. K. Patel, and D. F. Price, "Efficient pumping schemes for high average brightness collisional x-ray lasers," Proc. SPIE 5197, 213-219 (2003).

4. W. Kruer, The Physics of Laser Plasma Interactions (Westview, 2003).

5. M. Gruenig, C. Imesch, F. Staub, and J. E. Balmer, "Saturated $\mathrm{x}$-ray lasing in Ni-like $\mathrm{Sn}$ at $11.9 \mathrm{~nm}$ using the grazing incidence scheme," Opt. Commun. 282, 267-271 (2009).

6. D. Ursescu, "Grazing incidence pumped $\mathrm{Zr}$ x-ray laser for spectroscopy on Li-like ions," Ph.D. Thesis (J. Gutenberg University, 2006)

7. R. C. Elton, X-Ray Lasers (Academic, 1990)

8. G. J. Pert, "Optimizing the performance of nickel-like collisionally pumped x-ray lasers," Phys. Rev. A 73, 033809 (2006).

9. Y. Wang, M. A. Larotonda, B. M. Luther, D. Alessi, B. Berrill, V. N. Shlyaptsev, and J. J. Rocca, "Demonstration of highrepetition-rate tabletop soft-x-ray lasers with saturated output at wavelengths down to $13.9 \mathrm{~nm}$ and gain down to 10.9 nm," Phys. Rev. A 72, 053807 (2005). 